\title{
Interaction of prostate cancer and neurons - the neuro-neoplastic synapse
}

\author{
MJ Voss*, B Niggemann, KS Zaenker and F Entschladen
}

Address: University Witten/Herdecke, Immunology, Witten, Germany

* Corresponding author

from 12th Joint Meeting of the Signal Transduction Society (STS). Signal Transduction: Receptors, Mediators and Genes Weimar, Germany. 29-31 October 2008

Published: 26 February 2009

Cell Communication and Signaling 2009, 7(Suppl I):A40 doi:I0.II86/I478-8IIX-7-SI-A40

This abstract is available from: http://www.biosignaling.com/content/7/SI/A40

(c) 2009 Voss et al; licensee BioMed Central Ltd.

As the angiogenesis is well known for tumor tissue supplemented with nutrients and growth factors, the neoneurogenesis may play an important role in tumor development, too. Tumor cells respond to cytokines, chemokines, growth factors as well as neurotransmitters in for example increased migration and proliferation. It seems to be crucial for a tumor tissue to interact with its environment and obtain nutrients and signal substances, as well as the tumor release angiogenetic factors itself. In our work, we investigate the mutual influences of tumor cells and neural structures to elucidate the complex interaction and effect of cancer and metastasis forming within the body.

Cells of the prostate cancer cell line PC-3 release a broad range of signal substances e.g. nerve growth factor, brain derived neurotrophic factor, vascular endothelial growth factor A and C, as well as the cytokines IL-2 and IL-1 beta, which are key regulators of angiogenesis in cancer. We were able to show that IL- 2 and IL-1 beta strongly increase the migratory activity of human neuroblastoma cells SHSY5Y in our well-established 3D cell migration assay from $30 \%$ spontaneous locomotion to 60 and 50\% increase, respectively. In contrast, the migration activity of prostate cancer cells significant increases when exposed to the neurotransmitter dopamine and neuropeptide substance P up to $25 \%$. This indicates a close relation and impact of prostate cancer cells and neural structures as a complex association, not only as separate aggregation acting for its own. These cells affect each other by releasing there specific signal substances, which in turn can activate via receptor mediated cell signaling their locomotion and prolifera- tion attitude. Understanding the broad interaction of these cells during cancer and metastasis forming may help us to develop new approaches for cancer therapy in the future.

\section{Acknowledgements}

This work was supported by the Fritz-Bender Foundation (Munich, Germany). 